\title{
TECHNICAL NOTE: ASSAY OF CELL QUANTITY IN THE FIBROBLAST- POPULATED COLLAGEN MATRIX WITH A TETRAZOLIUM REAGENT
}

\author{
Mark A.Carlson \\ Department of Surgery, University of Nebraska Medical Center and the Omaha VA Medical Center, Omaha, NE, \\ USA
}

\begin{abstract}
Determination of cell quantity in three-dimensional culture systems, such as the fibroblast-populated collagen matrix, can be difficult secondary to the abundance of extracellular collagen. The WST-1 assay, which can quantitate cellular processes in monolayer culture, was tested as a measure of relative cell quantity in a three-dimensional culture system, the fibroblast-populated collagen matrix. The assay had acceptable accuracy and precision, and was not affected by the extracellular matrix. It was concluded that the assay was a reasonable alternative to other measures of cell quantity in the fibroblast-populated collagen matrix.
\end{abstract}

Key words: fibroblast, collagen, matrix, lattice, wound healing, WST-1, tetrazolium, cellular assay

\section{Address for correspondence: \\ Mark A. Carlson \\ Surgery 112, VA Medical Center \\ 4101 Woolworth Avenue \\ Omaha, NE 68105, USA}

Tel: 402-346-8800, ext. 5371

Fax: 402-977-5672

E-mail: macarlso@unmc.edu

\section{Introduction}

One intention of three dimensional culture systems is to model in vivo conditions more accurately than traditional two-dimensional (i.e., monolayer) systems (Cukierman et al., 2002). The fibroblast-populated collagen matrix (or lattice) has been utilized as model of either skin or granulation tissue, depending on culture conditions (Bell et al., 1981; Carlson and Longaker, 2004). Immunoblotting commonly is used in the fibroblastpopulated collagen matrix to assay specific protein levels. As is the case in monolayer systems, the relevance of immunoblotting depends in part on quantitative protein loading of each lane in the electrophoretic gel. With monolayer cells, this loading commonly is equilibrated by the determination of protein concentration in each cellular lysate of a given experiment. Protein assays (e.g., Lowry (Lowry et al., 1951) or Bradford-based (Bradford, 1976) assays) typically are not employed to determine protein concentration in a lysate of the fibroblastpopulated collagen matrix, however, because the overabundance of collagen in such a lysate would render a protein assay inaccurate.

As an alternative to the protein assay, the "loading control" (i.e., an immunoblot of an abundant cellular protein such as such as $\alpha$-tubulin or actin) often is used to demonstrate equivalent protein loading of immunoblot lanes. An immunoblot loading control assumes, among other things, that the level of the control protein is not affected by divergent experimental conditions. In the fibroblast-populated collagen matrix, however, it is not entirely clear whether the levels of abundant proteins remain constant during matrix contraction; this process involves extensive cytoskeletal remodelling (Carlson and Longaker, 2004). So it would be somewhat useful under these circumstances to have an alternative method that could replace or supplement the protein assay or the loading control for normalization of lane loading.

The WST-1 assay is a possible alternative method to normalize cellular lysates derived from the fibroblastpopulated collagen matrix. Cleavage of the WST-1 reagent (water-soluble tetrazolium; 4-[3-(4-iodophenyl)-2-(4nitrophenyl)-2H-5-tetrazolio]-1,3-benzene disulfonate) by ubiquitous plasma membrane enzymes of live cells yields an easily-quantified color change which is proportional to the mass of living cells (Berridge et al., 2005; Ishiyama et al., 1996). The WST-1 assay is well-described in monolayer systems for assay of various metabolic activities and cellular proliferation (Berridge et al., 2005), but there are few publications which refer to this assay in three-dimensional systems such as the fibroblastpopulated collagen matrix (Daniels et al., 2003) or banked skin (Alotto et al., 2002). Specifically, the use of the WST1 assay for determination of relative cell quantity in the 
fibroblast-populated collagen matrix has not been described. In the following short report we provide a detailed analysis of the WST-1 assay performance in this three-dimensional culture system.

\section{Materials and Methods}

\section{Cell culture and collagen matrices}

Primary human dermal fibroblasts were cultured from explants of neonatal foreskin obtained at the time of circumcision from our newborn nursery. The use of discarded human tissue was approved by our Institutional Review Board. Cells were maintained in T75 flasks with 10\% FBS (Invitrogen, Carlsbad, CA, USA) in DMEM (growth medium) as previously described (Grinnell et al., 1999). Collagen matrices were prepared as previously described (Grinnell et al., 1999); each matrix contained 200,000 cells (passage 9) in an initial volume of $200 \mu \mathrm{l}$ $\left(10^{6} \mathrm{cell} / \mathrm{ml}\right)$ with a $1.5 \mathrm{mg} / \mathrm{ml}$ of collagen (Vitrogen 100 ; Cohesion Technologies; Angiotech, Vancouver, Canada), and was cultured in growth medium supplemented with vitamin $\mathrm{C}(50 \mu \mathrm{g} / \mathrm{ml})$. Cell counts were performed in triplicate with a haemocytometer.

\section{Assays}

The DNA assay was adopted from a standard protocol (Sambrook and Russell, 2001). Lysates were clarified with centrifugation $\left(20,000 \mathrm{~g}, 30 \mathrm{~min}, 4^{\circ} \mathrm{C}\right)$, and then supernatant $(10 \mu \mathrm{l})$ was added to $5 \mathrm{ng} / \mathrm{ml}$ bisbenzimide (Hoechst 33258; Sigma, St. Louis, MO, USA) in PBS (final volume $2 \mathrm{ml}$; performed in triplicate). Fluorescence was read on a spectrophotometer (Perkin Elmer L550B; Perkin Elmer, Wellesley, MA, USA) using excitation/emission settings of $356 / 458 \mathrm{~nm}$; sample concentration was determined against DNA standards of $0-1 \mu \mathrm{g} / \mathrm{ml}$. The WST1 assay (Cell Proliferation Reagent WST-1; Roche Applied Science, Basel, Switzerland) was performed as per the manufacturer's instructions. Unless otherwise specified, $20 \mu \mathrm{l}$ of reagent was added to a $1.0 \mathrm{ml}$ volume of growth medium containing a single fibroblast-populated collagen matrix, as described above. Absorbance (420-480 nm) of the medium was read on a Perkin Elmer L550B spectrophotometer. Immunoblotting was performed as previously described (Carlson et al., 2004). The antiGAPDH antibody was obtained from Abcam (Cambridge, UK) (ab9485). A Lowry-based (Lowry et al., 1951) protein assay (DC Protein Assay; Bio-Rad, Hercules, CA; USA) was used as per the manufacturer's instructions. Matrix cell counts were performed by first digesting the matrix with a trypsin/collagenase sequence (Grinnell et al., 1999), spinning the intact cells down, resuspending them in DMEM, and then counting in triplicate with a haemocytometer.

\section{Statistics}

Numerical data are reported as the mean \pm standard deviation. Groups of unpaired numerical data were compared with analysis of variance (ANOVA) with a level of significance $p<0.05$. Each experiment was performed 2-5 times.

\section{Results and Discussion}

It was confirmed that a typical Lowry-based protein assay was influenced by the presence of collagen at concentrations found in lysates of the fibroblast-populated collagen matrix (Figure $1 \mathrm{~A}$ ). In fact, the protein assay was about twice as sensitive to collagen as it was to the albumin standards. So a direct colorimetric measure of cellular protein in a collagen matrix lysate would be meaningless, since the background collagen signal would overwhelm signal from the cellular protein. This problem might be circumvented by isolating the fibroblasts with centrifugation after matrix digestion with collagenase (MMP-1), but this is a 30-60 min process during which intact cells are subjected to harsh conditions that could influence immunoblot results, such as with kinase phosphorylation. In search of a more useful assay of "cell quantity," absorbance spectra of media derived from fibroblast-populated collagen matrices treated with WST1 (Figure 1B) were performed; an absorbance peak between 420 and $480 \mathrm{~nm}$ was observed (consistent with the manufacturer's specifications). Matrices without cells did not yield a peak. Dose-effect studies of both reagent (Figure 1C) and time (Figure 1D) were ran next in order to determine optimal assay conditions in the fibroblastpopulated collagen matrix. With a constant assay incubation period, there was a linear relationship between peak absorbance and added WST-1 reagent (up to $40 \mu \mathrm{l}$ ). With a constant WST-1 aliquot, the absorbance increased linearly with incubation time, out to $4 \mathrm{hr}$. Based on this data, final assay conditions for a single matrix (containing $\sim 2 \times 10^{5}$ fibroblasts) in $1.0 \mathrm{ml}$ of medium became $20 \mu \mathrm{l}$ (WST-1 aliquot) and $2 \mathrm{hr}$ (incubation at $37^{\circ} \mathrm{C}$ ). In addition, media samples from Figure 1D were stored at $4^{\circ} \mathrm{C}$ for 24 $\mathrm{hr}$, and the re-read absorbances indicated no change from initial values. So assay media can be stored for short periods without affecting absorbance spectra.

In order to determine how well the WST-1 assay correlated with other measures of cell quantity in the fibroblast-populated collagen matrix, four groups of matrices seeded with different cell numbers were incubated for three days and then assayed with WST-1, manual cell counts, DNA concentration, and GAPDH immunoblot densitometry (Figure 2). The results of each assay were plotted against the results of each of the other three assays, and the resultant correlation coefficient for each of these plots (total of six pair wise combinations) were all $>0.9$. This result demonstrated reasonable agreement among these assays of cell quantity in groups of collagen matrices with varying cell number. It appeared that the WST-1 assay provided a fairly accurate determination of cell quantity in this three-dimensional culture system. Another assay of cell quantity which has been employed in the fibroblastpopulated collagen matrix is lysate LDH activity (Fringer and Grinnell, 2003; Korzeniewski and Callewaert, 1983); however, this assay had marginal precision in the author's laboratory, and thus was not used for this work.

Another performance test of the WST-1 assay in the fibroblast-populated collagen matrix was done using typical experimental conditions of mechanical loading and unloading. Under conditions of mechanical loading 


\section{A}
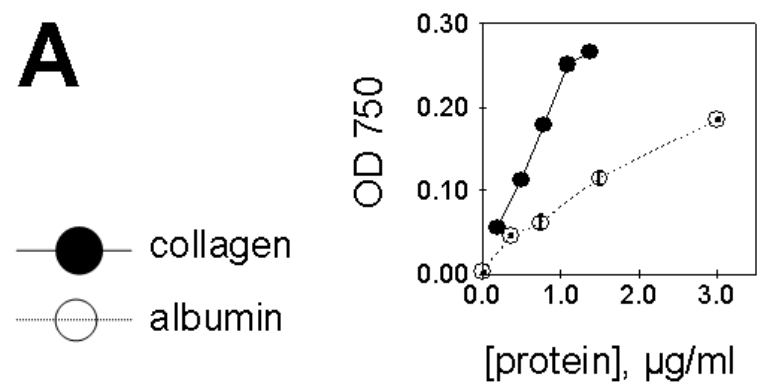

B

C

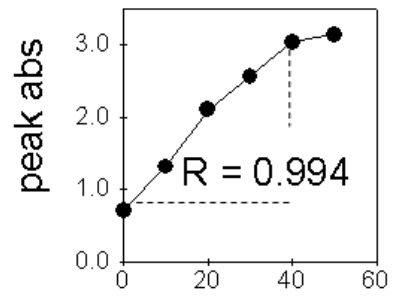

$\mu \mathrm{W}$ WST-1
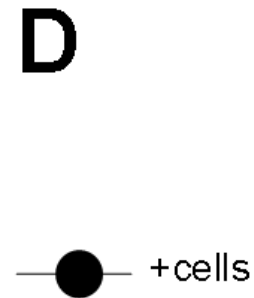

+ + cells SM

....-. cell-free

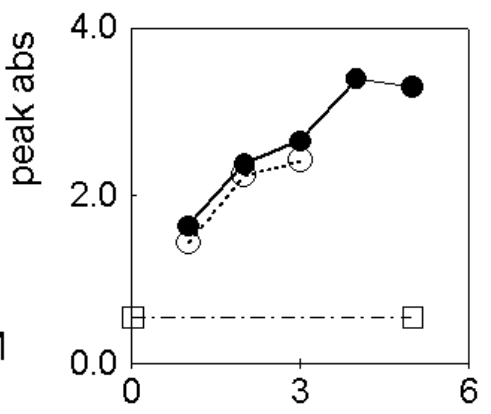

WST-1 incubation (hr)
A

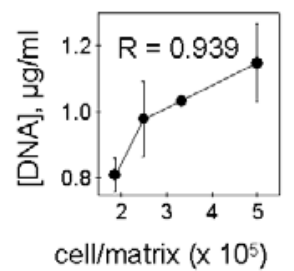

GAPDH
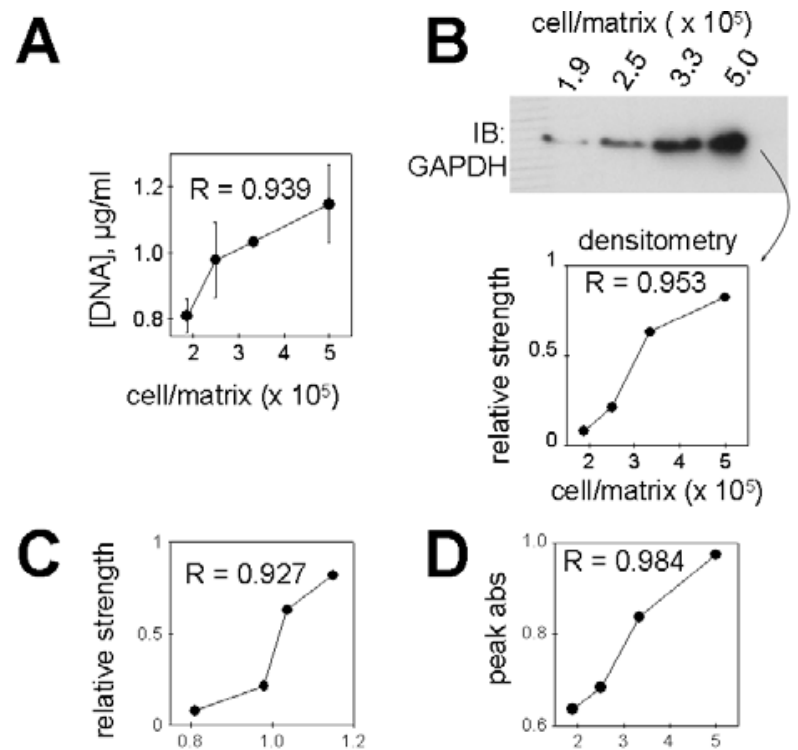

D

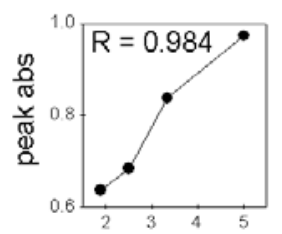

[DNA], $\mu \mathrm{g} / \mathrm{ml}$
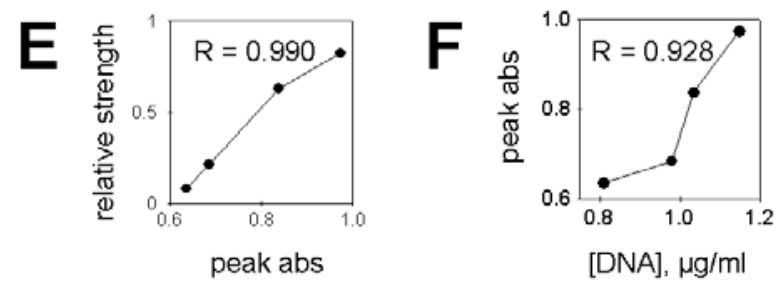

Figure 2. WST-1 assay compared to cell counts, DNA concentration and immunoblot densitometry in the fibroblast-populated collagen matrix. Four groups of matrices seeded with 50,000-200,000 cell/matrix were incubated in growth medium for $72 \mathrm{hr}$, and then processed for DNA concentration, cell counting, GAPDH immunoblot densitometry, and the WST-1 assay as detailed in the Methods. The data from each assay was plotted pairwise against the data from each of the other three assays. The correlation coefficient (R) was determined from each paired plot. (A) DNA concentration (in $\mu \mathrm{g} / \mathrm{ml}$ ) vs. cell counting (in cell/ matrix). (B) Immunoblot densitometry (expressed as relative strength with a range of $0-1$ ) vs. cell counting. (C) Immunoblot densitometry vs. DNA concentration. (D) WST-1 assay (expressed as peak absorbance between 420-480 nm) vs. cell counting. (E) Immunoblot densitometry vs. the WST-1 assay. (F) WST-1 assay vs. DNA concentration.

Figure 1. Rationale for and basics of WST-1 assay. (A) Comparison of collagen and albumin in a Lowry-based (Lowry et al., 1951) protein assay (Bio-Rad DC). OD $750=$ optical density at light wavelength of $750 \mathrm{~nm}$. (B) Typical absorbance spectrum for the WST-1 assay. Fibroblast-populated collagen matrices were cultured for $72 \mathrm{hr}$ in growth medium, and then WST-1 $(10 \mu \mathrm{l}$ ) was added (assay volume $=1,000 \mu \mathrm{l}$ ); media was removed for spectrophotometry $60 \mathrm{~min}$ after WST-1 addition. Shaded area = wavelength range $(420-480 \mathrm{~nm})$ in which absorbance peak was measured. (C) Response of WST-1 assay to substrate quantity. Attached fibroblast-populated collagen matrices were cultured for $72 \mathrm{hr}$; WST-1 then was added as indicated, and absorbance spectra were measured $2 \mathrm{hr}$ later. Correlation coefficient of the left-most four data points is shown. (D) Response of WST-1 assay to assay incubation time. Attached fibroblast-populated collagen matrices were cultured for $72 \mathrm{hr}$; WST-1 (20 $\mu \mathrm{l})$ then was added, and the absorbance spectra were measured at the indicated times post-WST-1 addition. SM = stored media (i.e., samples of media which yielded the closed circle plot were stored at $4^{\circ} \mathrm{C}$ for $24 \mathrm{hr}$ and then re-read in the spectrophotometer). 

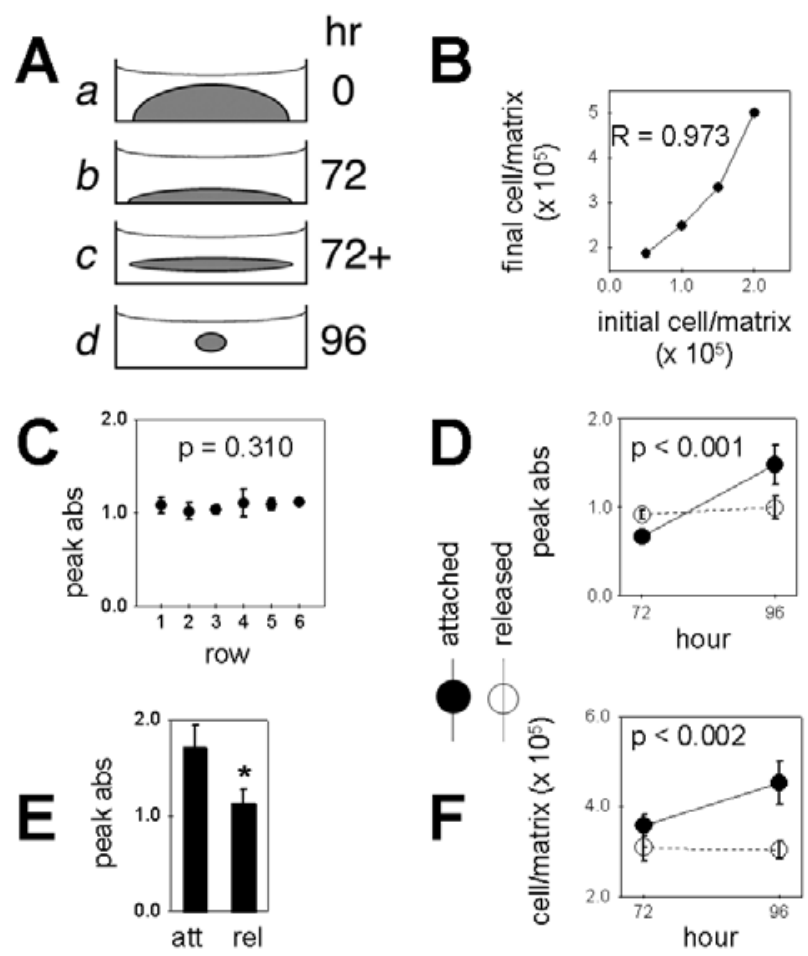

Figure 3. The WST-1 assay reflects known biology of the fibroblast-populated collagen matrix. (A) Schematic of the model. (a) A solution of bovine collagen and human neonatal foreskin fibroblasts is pipeted into a culture well and subsequently polymerizes into a gel; growth medium then is added. (b) The matrix, which is attached (anchored) to the tissue culture plastic, is incubated for $72 \mathrm{hr}$; the fibroblasts within the matrix generate mechanical tension. (c) The matrix then is physically detached (using a spatula) from the floor of the culture well; sudden loss of anchorage results in cell-driven matrix contraction. (d) Appearance of matrix $24 \mathrm{hr}$ after detachment (or $96 \mathrm{hr}$ after set-up) with the attendant contraction. $(\boldsymbol{B})$ Relationship between initial $(\mathrm{t}=0)$ and final $(\mathrm{t}=72 \mathrm{hr})$ cell counts in attached matrices incubated in growth medium. Correlation coefficient is shown. (C) Precision of the WST-1 assay. Attached matrices (72 hr old) underwent the WST-1 assay as described in the Methods. Each data point represents the mean \pm sd of 6 wells (i.e., one row in a 24-well plate); plot represents data of 6 rows (not statistically different; ANOVA). (D) Effect of matrix release on the WST-1 assay after $24 \mathrm{hr}$. Attached matrices were incubated for $72 \mathrm{hr}$, and then half were released; the WST-1 assay was performed $24 \mathrm{hr}$ later (ANOVA result shown). (E) Immediate effect of matrix release on the WST-1 assay. Attached matrices (72 hr old) were \pm released, and the WST-1 assay was performed immediately. (F) Same as $\boldsymbol{D}$, but cell counts were measured.

(resulting from matrix attachment to the culture dish; Figure $3 \mathrm{~A}$, panel $b$ ) and serum supplementation, fibroblasts in the collagen matrix have a proliferative phenotype and modulate protein synthesis and secretion (Carlson and Longaker, 2004). This effect on proliferation was reproduced in Figure 3B, in which initial (seeding) vs. final matrix cell counts are plotted, demonstrating an approximate population doubling after $72 \mathrm{hr}$ of matrix attachment at each seeding number. If the mechanical loading is removed (Figure 3A, c-d), the fibroblasts in the collagen matrix become quiescent and some undergo apoptosis (Carlson and Longaker, 2004). This effect on fibroblast biology was reproduced in Figure $3 \mathrm{~F}$, in which the cell count from detached matrices decreased slightly while the count in the attached matrices continued to increase. When the WST-1 assay was applied to a similar experiment (Figure 3D), a result analogous to the cell counts was obtained; that is, the peak absorbance continues to increase under mechanical loading (attachment) of the fibroblast-populated collagen matrix, while unloading (detachment) of the mechanically loaded matrix resulted in a leveling off of the absorbance curve. It was concluded that the WST-1 assay accurately reflected the known biology of the fibroblast-populated collagen matrix under varying mechanical load.

There was a concern that acute matrix detachment by itself would influence the WST-1 assay, perhaps by some shearing effect on the plasma membrane (Lin et al., 1997). In one experiment (Figure 3E) we noted an acute decrease in the WST-1 assay immediately after matrix detachment, which suggested that the simple act of detachment might affect enzymatic conversion of the WST-1 reagent. This acute effect of detachment, however, could not be reproduced, and subsequently it was doubtful if this was a real effect. In some experiments there was a small (statistically insignificant) increase in WST-1 conversion associated with acute matrix detachment (for example, see the $72 \mathrm{hr}$ time point in Figure 3D). These results then raised a concern regarding the precision of the WST-1 assay; in order to address this, 24-well plates of identical attached matrices were organized by row and column and run through the WST-1 assay (a representative experiment is shown in Figure 3C). The results of the WST-1 assay did not vary significantly from matrix to matrix. So in addition to being reasonably accurate, the WST-1 assay also is reasonably precise in the fibroblast-populated collagen matrix.

The exclusive cell type utilized in this paper was the human neonatal foreskin fibroblast. Since the WST-1 assay has worked well with a variety of cell types in monolayer culture (Berridge et al., 2005), and because the assay worked well with fibroblasts embedded in collagen in the present paper, it is probable that the assay would work well with other fibroblasts, smooth muscles cells, and the many other cell types which have been embedded in collagen (Grinnell, 2003). The exclusive extracellular matrix material utilized in this paper was type I bovine collagen. It is probable that other matrix materials (such as fibrin, fibronectin, or other collagens) would be compatible with this assay, but his has not been demonstrated. One potential problem when using any three-dimensional matrix is binding/sequestration of ligands (such as peptides, lipids, or other small molecules) by the matrix (Allan et al., 1995). Such binding might provide a "sink" for the ligand, thereby influencing experimental results. There was no consistent evidence of a ligand sequestration in the experiments of this paper. With regard to matrix porosity, the interstices of the 
hydrated collagen matrix (as determined with electron microscopy) are on the order of $1 \mu \mathrm{m}$ (Elsdale and Bard, 1972; Lorimier et al., 1998). It is unlikely that pores of this size would inhibit diffusion of small molecules. Some macromolecules (such as inulin) have variable diffusion across experimental collagen matrices with differing ultrastructure (Gilbert and Kim, 1990). Although a separate determination was not performed, there was no evidence of a reagent diffusion problem in the experiments of this paper.

The WST-1 assay performed acceptably as a measure of "cell quantity" in the three-dimensional fibroblast culture system. Whether the actual entity that this assay quantified was more a measure of cell volume vs. membrane area vs. cell mass vs. something else is debatable, but perhaps not as relevant. The advantages of the WST-1 assay are that it (i) can be performed on live cells without obvious perturbation in their biology, (ii) is not influenced by an excess of extracellular matrix protein, (iii) is simple to perform, and (iv) has acceptable accuracy and precision. The main disadvantages are that it (i) is not a hard measure like $\mu \mathrm{g}$ of protein or DNA (so the WST-1 assay only yields relative quantitation), and (ii) it requires a 1-2 hr incubation just prior to the experimental endpoint. For purposes such as normalization of lane loading for immunoblots, however, the WST-1 assay is a reasonable alternative to the determination of lysate protein concentration, which is not practical in the fibroblastpopulated collagen matrix.

\section{Acknowledgments}

The authors would like to acknowledge the technical assistance of Chris Hansen and Amy Prall. Supported by a grant from the NIH (K08 GM00703) to MAC.

\section{References}

Allan JA, Docherty AJ, Barker PJ, Huskisson NS, Reynolds JJ, Murphy G (1995) Binding of gelatinases A and B to type-I collagen and other matrix components. Biochem J 309: 299-306.

Alotto D, Ariotti S, Graziano S, Verrua R, Stella M, Magliacani G, Castagnoli C (2002) The role of quality control in a skin bank: tissue viability determination. Cell Tissue Bank 3: 3-10.

Bell E, Ehrlich HP, Buttle DJ, Nakatsuji T (1981) Living tissue formed in vitro and accepted as skinequivalent tissue of full thickness. Science 211: 1052-1054.

Berridge MV, Herst PM, Tan AS (2005) Tetrazolium dyes as tools in cell biology: New insights into their cellular reduction. Biotechnol Annu Rev 11: 127-152.

Bradford MM (1976) A rapid and sensitive method for the quantitation of microgram quantities of protein utilizing the principle of protein-dye binding. Anal Biochem 72: 248-254.
Carlson MA, Longaker MT (2004) The fibroblastpopulated collagen matrix as a model of wound healing: a review of the evidence. Wound Repair Regen 12: 134147.

Carlson MA, Longaker MT, Thompson JS (2004) Modulation of FAK, Akt, and p53 by stress release of the fibroblast-populated collagen matrix. J Surg Res 120: 171177.

Cukierman E, Pankov R, Yamada K (2002) Cell interactions with three-dimensional matrices. Curr Opin Cell Biol 14: 633.

Daniels JT, Cambrey AD, Occleston NL, Garrett Q, Tarnuzzer RW, Schultz GS, Khaw PT (2003) Matrix metalloproteinase inhibition modulates fibroblast-mediated matrix contraction and collagen production in vitro. Invest Ophthalmol Vis Sci 44: 1104-1110.

Elsdale T, Bard J (1972) Collagen substrata for studies on cell behavior. J Cell Biol 54: 626-637.

Fringer J, Grinnell F (2003) Fibroblast quiescence in floating collagen matrices: decrease in serum activation of MEK and Raf but not Ras. J Biol Chem 278: 2061220617.

Gilbert DL, Kim SW (1990) Macromolecular release from collagen monolithic devices. J Biomed Mater Res 24: 1221-1239.

Grinnell F (2003) Fibroblast biology in threedimensional collagen matrices. Trends Cell Biol 13: 264269.

Grinnell F, Zhu M, Carlson MA, Abrams JM (1999) Release of mechanical tension triggers apoptosis of human fibroblasts in a model of regressing granulation tissue. Exp Cell Res 248: 608-619.

Ishiyama $\mathrm{M}$, Tominaga $\mathrm{H}$, Shiga $\mathrm{M}$, Sasamoto $\mathrm{K}$, Ohkura Y, Ueno K (1996) A combined assay of cell viability and in vitro cytotoxicity with a highly watersoluble tetrazolium salt, neutral red and crystal violet. Biol Pharm Bull 19: 1518-1520.

Korzeniewski C, Callewaert DM (1983) An enzymerelease assay for natural cytotoxicity. J Immunol Methods 64: 313-320.

Lin YC, Ho CH, Grinnell F (1997) Fibroblasts contracting collagen matrices form transient plasma membrane passages through which the cells take up fluorescein isothiocyanate-dextran and $\mathrm{Ca} 2+$. Mol Biol Cell 8: 59-71.

Lorimier S, Hornebeck W, Godeau G, Pellat B, Gillery P, Maquart FX, Laurent-Maquin D (1998) Morphometric studies of collagen and fibrin lattices contracted by human gingival fibroblasts; comparison with dermal fibroblasts. J Dent Res 77: 1717-1729.

Lowry OH, Rosebrough NJ, Farr AL, Randall RJ (1951) Protein measurement with the Folin phenol reagent. J Biol Chem 193: 265-275.

Sambrook J, Russell DW (2001) Molecular Cloning: A Laboratory Manual. Cold Spring Harbor Laboratory Press, Cold Spring Harbor. 